\title{
Microsurgical and endoscopic approaches to the pulvinar
}

\author{
Osamu Akiyama, MD,1,2 Ken Matsushima, MD, 1,3 Abuzer Gungor, MD,,4 Satoshi Matsuo, MD, 1,5 \\ Dylan J. Goodrich, BS, ${ }^{6}$ R. Shane Tubbs, MS, PA-C, PhD, ${ }^{6,7}$ Paul Klimo Jr., MD, MPH, ${ }^{8}$ \\ Aaron A. Cohen-Gadol, MD, MSc, ${ }^{9}$ Hajime Arai, MD, ${ }^{2}$ and Albert L. Rhoton Jr., MD1
}

\begin{abstract}
${ }^{1}$ Department of Neurological Surgery, University of Florida, Gainesville, Florida; ${ }^{2}$ Department of Neurosurgery, Juntendo University, Tokyo; ${ }^{3}$ Department of Neurosurgery, Tokyo Medical University, Tokyo, Japan; ${ }^{4}$ Department of Neurosurgery, Bakirkoy Research and Training Hospital for Neurology, Neurosurgery, and Psychiatry, Istanbul, Turkey; ${ }^{5}$ Department of Neurosurgery, Graduate School of Medical Sciences, Kyushu University, Fukuoka, Japan; ${ }^{6}$ Department of Anatomical Sciences, St. George's University, St. George's, Grenada; ${ }^{7}$ Seattle Science Foundation, Seattle, Washington; ${ }^{8}$ Semmes-Murphey Neurologic \& Spine Institute and Le Bonheur Children's Hospital, Memphis, Tennessee; and ${ }^{9}$ Goodman Campbell Brain and Spine, Department of Neurological Surgery, Indiana University School of Medicine, Indianapolis, Indiana
\end{abstract}

OBJECTIVE Approaches to the pulvinar remain challenging because of the depth of the target, surrounding critical neural structures, and complicated arterial and venous relationships. The purpose of this study was to compare the surgical approaches to different parts of the pulvinar and to examine the efficacy of the endoscope as an adjunct to the operating microscope in this area.

METHODS The pulvinar was examined in 6 formalin-fixed human cadaveric heads through 5 approaches: 4 above and 1 below the tentorium. Each approach was performed using both the surgical microscope and $0^{\circ}$ or $45^{\circ}$ rigid endoscopes.

RESULTS The pulvinar has a lateral ventricular and a medial cisternal surface that are separated by the fornix and the choroidal fissure, which wrap around the posterior surface of the pulvinar. The medial cisternal part of the pulvinar can be further divided into upper and lower parts. The superior parietal lobule approach is suitable for lesions in the upper ventricular and cisternal parts. Interhemispheric precuneus and posterior transcallosal approaches are suitable for lesions in the part of the pulvinar forming the anterior wall of the atrium and adjacent cisternal part. The posterior interhemispheric transtentorial approach is suitable for lesions in the lower cisternal part and the supracerebellar infratentorial approach is suitable for lesions in the inferior and medial cisternal parts.

The microscope provided satisfactory views of the ventricular and cisternal surfaces of the pulvinar and adjacent neural and vascular structures. The endoscope provided multi-angled and wider views of the pulvinar and adjacent structures.

CONCLUSIONS A combination of endoscopic and microsurgical techniques allows optimal exposure of the pulvinar. https://thejns.org/doi/abs/10.3171/2016.8.JNS16676

KEY WORDS arteriovenous malformation; brain tumor; endoscope; pulvinar; surgical approaches; anatomy

$\mathrm{T}$ HE pulvinar, the largest and most posterior thalamic nucleus, is one of the most inaccessible sites in the brain. It can be the site of pathological lesions such as cavernous and arteriovenous malformations and gliomas. ${ }^{7,14,19,34,43}$ The pulvinar is located adjacent to the interhemispheric fissure, partially in the lateral ventricle and partially in the cisterns in the posterior part of the tentorial incisura. Operative indications for approaching the pulvinar are a matter of debate, and surgical approaches are challenging because of the complex anatomy of the area. ${ }^{27,36}$ However, well-circumscribed gliomas and hemorrhagic arteriovenous and cavernous malformations may be reasonable operative candidates. ${ }^{14,43}$ The endoscope has been reported to have advantages over the microscope in such deep exposures, including a smaller craniotomy, less dissection, minimal retraction, and more highly magnified focal exposures. . $^{8,13,21,23,26,36}$ Despite these advantages, there are no reports of endoscopic approaches to the pulvinar. 


\section{Methods}

The anatomy and surgical approaches around the pulvinar were examined in 6 formalin-fixed adult human cadaveric heads injected with colored silicone. Approaches above the tentorium include the superior parietal lobule, posterior interhemispheric transprecuneus, posterior interhemispheric transcallosal, and posterior interhemispheric transtentorial approaches. The visible neural and vascular structures related to the reachable parts of the pulvinar were examined in all approaches with the surgical microscope and rigid endoscope. In the study, the pulvinar is divided into 2 parts: ventricular and cisternal, separated by the fornix and choroidal fissure. The microscopic dissection was performed with the $3 \times$ to $40 \times$ magnifications provided by the operating microscope (Carl Zeiss AG).

The endoscopic dissection was performed with $0^{\circ}$ and $45^{\circ}$ angle, $4-\mathrm{mm}$-diameter optics and $30^{\circ}$ angle, $2.7-\mathrm{mm}$ diameter optics, 18-cm Hopkins endoscopes (Karl Storz $\mathrm{GmbH} \& \mathrm{Co}$ ), fixed with an endoscope holder, connected to a xenon light source and a high-definition camera.

\section{Results \\ Microsurgical Anatomy of the Pulvinar}

The pulvinar has a lateral ventricular surface and a surface that faces the quadrigeminal and ambient cisterns, which are separated by the crus of the fornix and the choroidal fissure, which surround the superior, posterior, and inferior surfaces of the pulvinar (Figs. 1-3). The cisternal part is divided into an upper and a lower part. Supra- or infratentorial surgical approaches to the pulvinar can be selected depending on the site of the lesion.

The thalamus is an ovoid nuclear mass located in the tentorial incisura between the basal ganglia and midbrain (Figs. 1-3). ${ }^{31}$ The pulvinar bulges posteriorly from the thalamus into the atrium and adjacent part of the body and temporal horn of the lateral ventricle and into the quadrigeminal cistern. The anterosuperior limit of the pulvinar lies along an imagined horizontal line extending vertically along the anterior edge of the pineal gland (Fig. 2A). The anteroinferior limit is at the anterior edge of the lateral and medial geniculate bodies. The posterior limb of the internal capsule and the striathalamic sulcus between the caudate nucleus and thalamus along which the stria terminalis passes form the lateral limit of the pulvinar. The medial and inferior surface of the pulvinar faces the ambient and quadrigeminal cisterns (Fig. 1C). The superior and inferior colliculi are located inferior and medial to the pulvinar (Figs. 2 and 3).

In approaching the pulvinars from posteriorly through the interhemispheric fissure, the posterior edge of the splenium will be encountered before the pulvinars, which are hidden below the splenium. Directing the approach anterior below the splenium will encounter the pineal gland in the midline on the medial side of the lower half of the paired pulvinars (Fig. 2B). The pulvinars bulge slightly posterior to the posterior tip of the pineal gland in the lateral part of the quadrigeminal cistern. Only a small inferomedial part of the cisternal surface of the pulvinar is exposed in the lateral wall of the quadrigeminal cistern.
The larger lateral part of the cisternal surface is hidden anterior to the occipital lobe and can be seen only with retraction. The fact that the splenium crosses both above and posterior to the pulvinar allows for a more superior approach directed along the superior surface of the splenium or an approach directed along a lower trajectory through the posterior splenium.

The crus of the fornix and choroidal fissure wrap around the upper, posterior, and lower surfaces of the pulvinar at the border between the cisternal and ventricular surfaces (Figs. 1, 2B, 3C, and 3D). The choroidal fissure is the narrow, $\mathrm{C}$-shaped cleft between the crus of the fornix and the thalamus, along which the choroid plexus is attached. The cisternal surface of the pulvinar faces the quadrigeminal cistern and can be exposed by opening the choroidal fissure if the approach is through the posterior body and atrium of the lateral ventricle. Another important relationship is to the calcarine sulcus, which extends forward with its anterior edge intersecting the junction between the isthmus of the cingulate and parahippocampal gyri at the level of the lower edge of the pulvinar. A cleft of the quadrigeminal cistern extends laterally between the anterior edge of the occipital lobe and the cisternal surface of the pulvinar to where the fornix wraps around the pulvinar at the border between the cisternal and ventricular surfaces. The junction of the parahippocampal gyri and the isthmus of the cingulate gyrus forms the posterior border of the cleft facing the cisternal part of the pulvinar (Figs. 1-3). This junction is formed where the anterior end of the cingulate sulcus cuts in and partially separates the cingulate and parahippocampal gyri. The posteromedial edge of the crus of the fornix extends posteriorly to blend into the occipital cortex at the area bordering the isthmus of the cingulate gyrus.

The posterior choroidal arteries pass first below, then posterior to and above the cisternal surface of the pulvinar and through the choroidal fissure at the level of the fimbria, crus, and body of the fornix to reach the choroid plexus in the temporal horn, atrium, and body of the lateral ventricle. The medial posterior choroidal artery arises from the posteromedial aspect of the proximal part of the posterior cerebral artery and passes around the cisternal part of the pulvinar to reach the choroid fissure and further ventrally (Fig. 3B). The lateral posterior choroidal artery originates from the posterior cerebral artery in the ambient and quadrigeminal cisterns and passes around the posteromedial surface of the pulvinar and through the choroidal fissure to terminate in the choroid plexus in the atrium and pulvinar (Fig. 3B).

The significant veins around the pulvinar are the internal cerebral, basal, and internal occipital veins, and other tributaries of the vein of Galen. The internal cerebral veins drain the deep parts of the hemisphere and course along the medial surface; the internal occipital vein crosses the posterior cisternal surface of the pulvinar to drain the anterior part of the area bordering the calcarine sulcus (Figs. $2 \mathrm{C}, 3 \mathrm{~B}$, and 3D). The posterior cerebral artery bifurcates into the parieto-occipital and calcarine arteries and courses around the midbrain below the pulvinar and through the lateral part of the quadrigeminal cistern. 


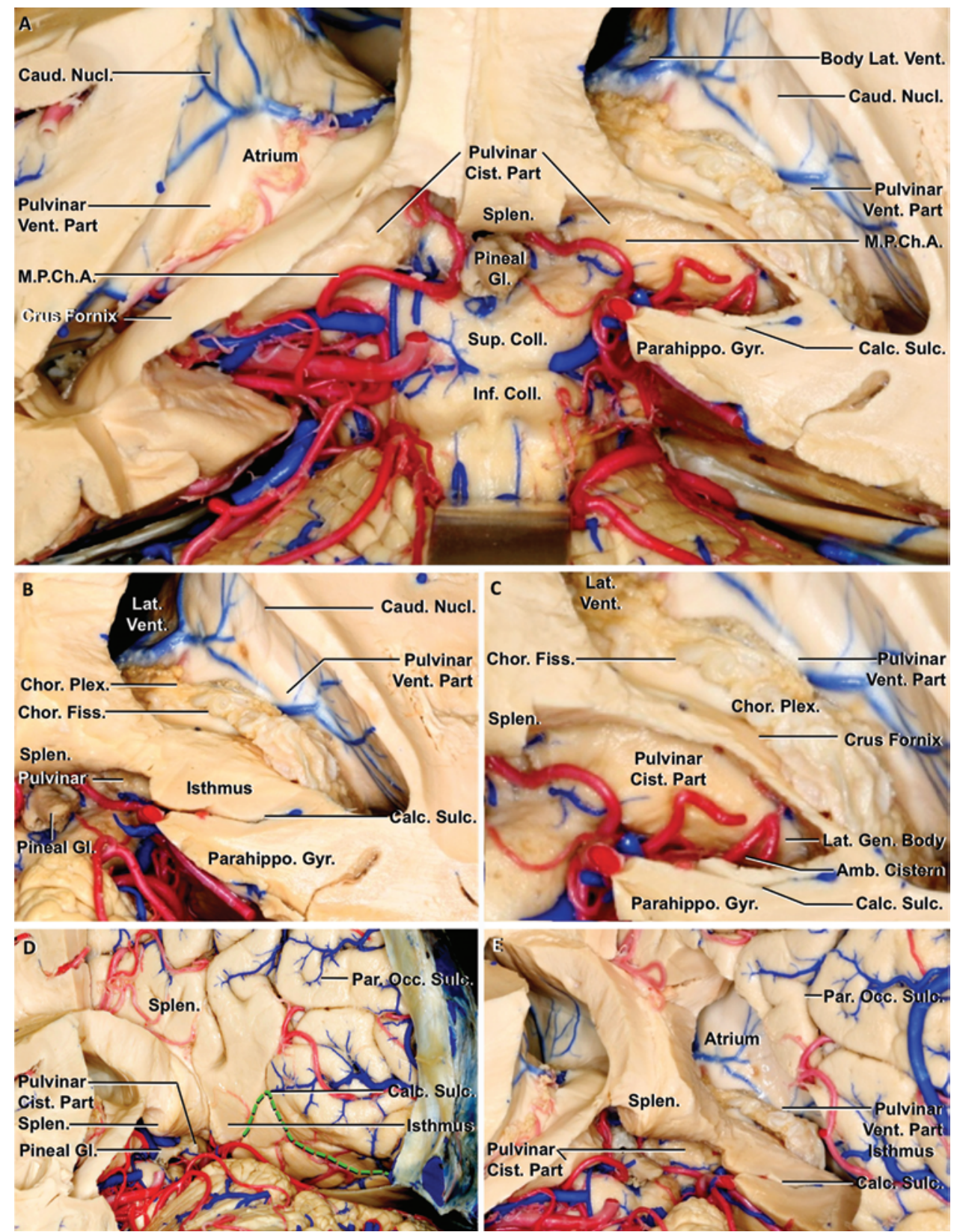

FIG. 1. Neural relationships of the pulvinar. A: Posterior view of a coronal section through the occipital lobes exposing the atria of the lateral ventricles and quadrigeminal cistern. Both the ventricular and cisternal parts of the pulvinar, separated by the crus of the fornix, have been exposed. The cisternal parts of the pulvinars have been exposed by removing the parahippocampal gyrus and isthmus of the cingulate gyrus. The pineal gland is located below the splenium. The superior colliculi is set inferomedial to the pulvinars. B and C: Stepwise exposure of the cisternal part of the right pulvinar. Coronal section through the right occipital lobe (B). The ventricular part of the pulvinar is exposed in the atrium. The cisternal part of the pulvinar is hidden by the crus of the fornix and isthmus of the cingulate gyrus. The anterior end of the calcarine sulcus extends forward between the parahippocampal gyrus and the isthmus. The posteromedial edge of the crus of the fornix blends into the isthmus of the cingulate gyrus. Coronal section through the right occipital lobe after removal of the area bordering the isthmus of the cingulate gyrus $(\mathrm{C})$. The pulvinar sits in the anterior wall of the atrium laterally and the quadrigeminal cistern medially. The lateral geniculate body, a component of the pulvinar, extends downward from the lower surface of the pulvinar into the roof of the ambient cistern. D and E: Stepwise exposures of the posteromedial part of the right hemisphere. The inferomedial edge of the cisternal part of the pulvinar is exposed to the right of the pineal gland. The calcarine sulcus extends forward from the occipital pole and along the lower edge of the pulvinar and pineal gland between the parahippocampal gyrus and the isthmus of the cingulate gyrus. The green dashed line extends along the calcarine sulcus (D). The medial surface of the occipital lobe has been opened to expose the atrium of the lateral ventricle (E). Amb. $=$ ambient; Calc. = calcarine; Caud. = caudate; Chor. = choroid, choroidal; Cist. = cisternal; Coll. = colliculus; Fiss. = fissure; Gen. = geniculate; Gl. = gland; Gyr. = gyrus; Inf. = inferior; Lat. = lateral; M.P.Ch.A. = medial posterior choroidal artery; Nucl. = nucleus; Par. Occ. = parieto-occipital; Parahippo. = parahippocampal; Plex. = plexus; Splen. = splenium; Sulc. = sulcus; Sup. = superior; Vent. $=$ ventricular, ventricle. 

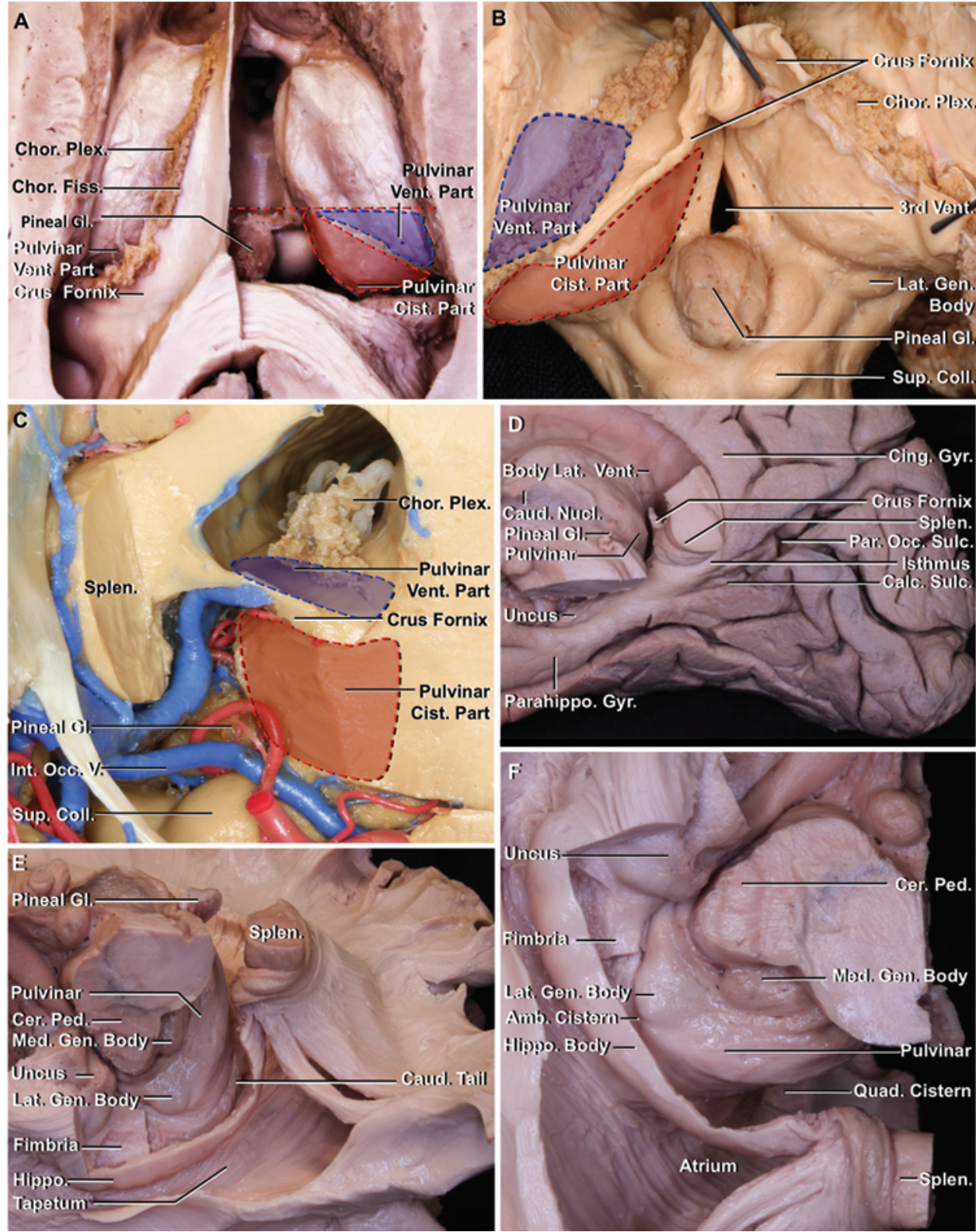

FIG. 2. Relationships of the pulvinar. A: Superior view with the fornix and choroid plexus preserved on the left side and removed on the right side. The pulvinars extend from the posterior part of the lateral ventricle and along the anterior wall of the atrium to the lower cisternal surface of the pulvinar, which includes the geniculate bodies. The ventricular part of the pulvinar (blue shaded area) is lateral to the choroidal fissure. The cisternal part of the pulvinar is hidden deep and medial to the fornix (red shaded area). B: Posterosuperior view of the pulvinars. The pineal gland is located between the lower part of the medial surface of the pulvinars, which overhang the superior colliculi. The right crus of the fornix has been divided and lifted away from the choroidal fissure, which marks the border between the cisternal (red shaded area) and the ventricular (blue shaded area) surfaces of the pulvinar. C: Posterolateral view, right pulvinar. Both the ventricular (blue shaded area) and cisternal (red shaded area) parts, separated by the crus of the fornix, are exposed. The internal cerebral and occipital veins have been exposed in the quadrigeminal cistern. D: Medial view of the right hemisphere. The body of the corpus callosum and the fornix have been removed. The crus of the fornix wraps around the atrial surface of the pulvinar. The pulvinar projects into the atrium and quadrigeminal cistern. The posterior part of the splenium of the corpus callosum crosses posterior to the pulvinar and pineal gland. The anterior end of the calcarine sulcus separates the isthmus of the cingulate and parahippocampal gyri at the posterior inferior edge of the pulvinar. E: Inferomedial view. The inferior edge of the pulvinar extends to the anterior edge of the lateral geniculate bodies. F: Inferior view. The anterior edge of the lateral geniculate body is at the lower lateral edge of the pulvinar. The pulvinar faces the quadrigeminal and ambient cisterns. Amb. = ambient; Calc. = calcarine; Caud. = caudate; Cer. = cerebellar, cerebral; Chor. = choroid, choroidal; Cing. = cingulate; Cist. = cisternal; Coll. = colliculus; Fiss. = fissure; Gen. = geniculate; GI. = gland; Gyr. = gyrus; Hippo. = hippocampus; Int. Occ. V. = internal occipital vein; Lat. = lateral; Med. = medial; Nucl. = nucleus; Par. Occ. = parieto-occipital; Parahippo. = parahippocampal; Ped. = peduncle; Plex. = plexus; Quad. = quadrigeminal; Splen. = splenium; Sulc. = sulcus; Sup. = superior; Vent. = ventricular, ventricle. 


\section{Approaches to the Pulvinar}

Superior Parietal Lobule Approach

The superior parietal lobule approach provides access to the surface of the pulvinar between the upper atrium and posterior body of the lateral ventricle and the superior part of the cisternal surface. The approach initially opens into the posterior part of the body of the lateral ventricle and adjacent part of the atrium and requires opening of the choroidal fissure, ipsilateral fornix, or the medial atrial surface just behind the fornix to reach the cisternal surface of the pulvinar (Fig. 4B and C). The posterior limb of the internal capsule borders the lateral edge of this exposure. In the three-quarter prone position with the face turned toward the floor so as to place the parietal area most superiorly, the dura is opened with the pedicle toward the superior sagittal sinus, and a cortical incision is directed along the superior parietal lobe to the posterior body and atrium (Fig. 4A). For endoscopy, the trajectory is the same as in the microscopic approach. A disadvantage of this approach is that it may cause visuospatial dysfunction in the nondominant hemisphere and aphasia in the dominant hemisphere because of the interruption of the optic radiations and speech pathways. ${ }^{17}$

Microscopic View. The first structures exposed are the surface of the pulvinar in the posterior body and atrium of the lateral ventricle, the choroid plexus, the choroidal fissure, and the crus of the fornix (Fig. 4B). To avoid injury to the fornix and to secure sufficient operative space, the choroidal fissure must be opened enough to allow dissection between the fornix and pulvinar anteriorly and laterally. Next, after retracting the fornix medially and posteriorly, the ipsilateral internal cerebral and basal veins, cisternal

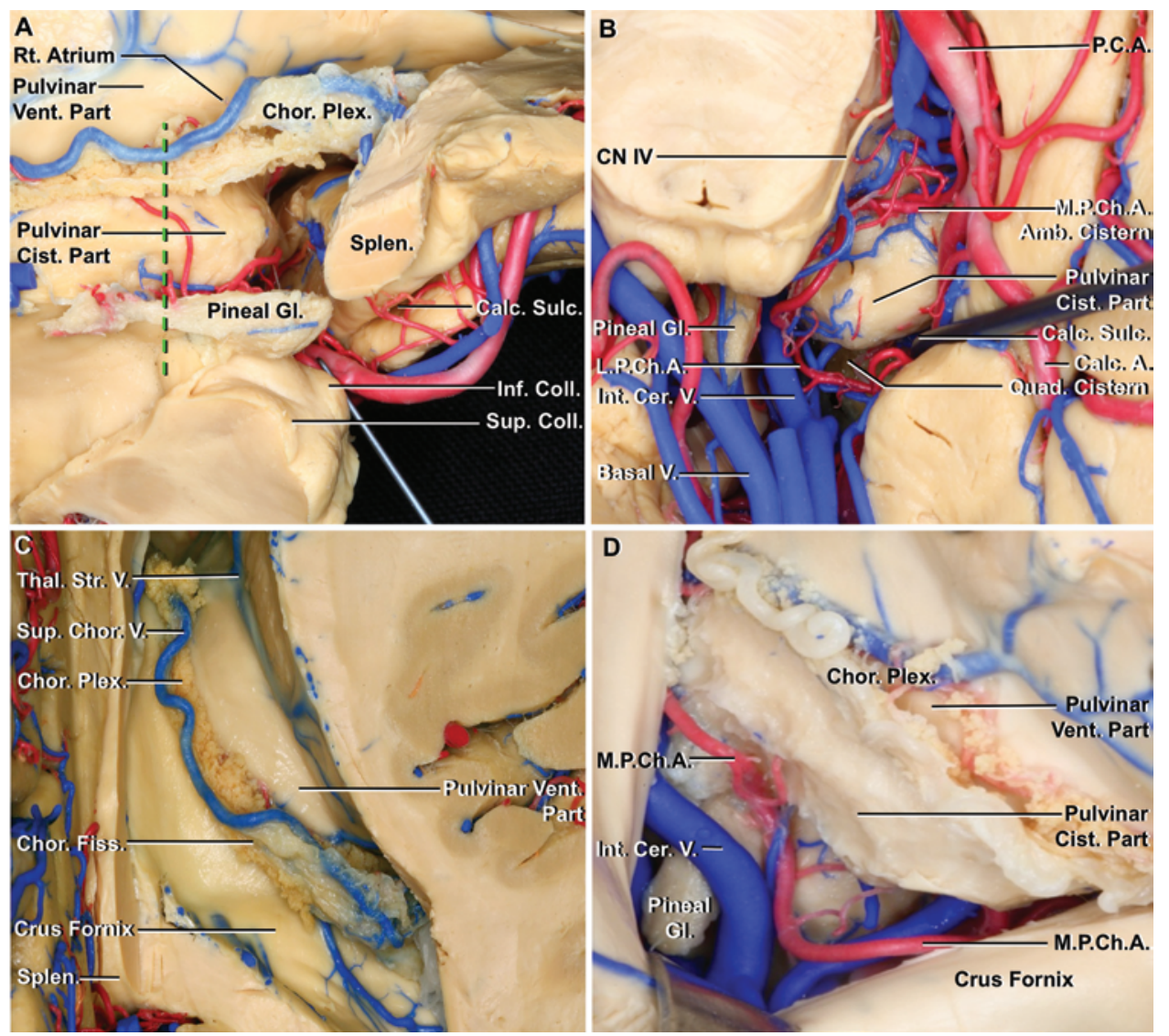

FIG. 3. Relationships of the pulvinar. A: Medial view. The right atrial and cisternal surfaces of the pulvinar have been exposed. The coronal plane along the anterior edge of the pineal gland (green dotted line) extends along the anterosuperior edge of the pulvinar. The pineal gland sits medial to the lower part of the pulvinar and anterior to the posterior edge of the splenium. B: Inferior view. The cisternal part of the pulvinar is exposed in the quadrigeminal and ambient cisterns. The calcarine branch of the posterior cerebral artery crosses the cistern. The right basal and left internal cerebral veins and medial and lateral posterior choroidal arteries, which arise from the posterior cerebral artery, are exposed. C: Superior view. Axial section of the right hemisphere at the level of the body of the lateral ventricle. The choroid plexus, crus of the fornix, and superior choroidal vein have been exposed. The crus of the fornix wraps around the posterior surface of the pulvinar and arches superomedially toward the splenium. The choroidal fissure is the narrow cleft between the crus of the fornix and the ventricular part of the pulvinar along which the choroid plexus is attached. D: Enlarged superior view. The choroidal fissure has been opened and the fornix has been displaced medially to expose the pineal gland, cisternal part of the pulvinar, and medial posterior choroidal artery. The internal cerebral vein passes medial to the pulvinar. $\mathrm{A}$. = artery; Amb. = ambient; Calc. = calcarine; Cer. = cerebral; Chor. = choroid, choroidal; Cist. = cisternal; Coll. = colliculus; Fiss. = fissure; GI. = gland; Inf. = inferior; Int. = internal; Lat. = lateral; L.P.Ch.A. = lateral posterior choroidal artery; M.P.Ch.A. = medial posterior choroidal artery; P.C.A. = posterior cerebral artery; Plex. = plexus; Quad. = quadrigeminal; Rt. = right; Splen. = splenium; Str. = striate; Sulc. = sulcus; Sup. = superior; Thal. = thalamo; V. = vein; Vent. = ventricular, ventricle. 


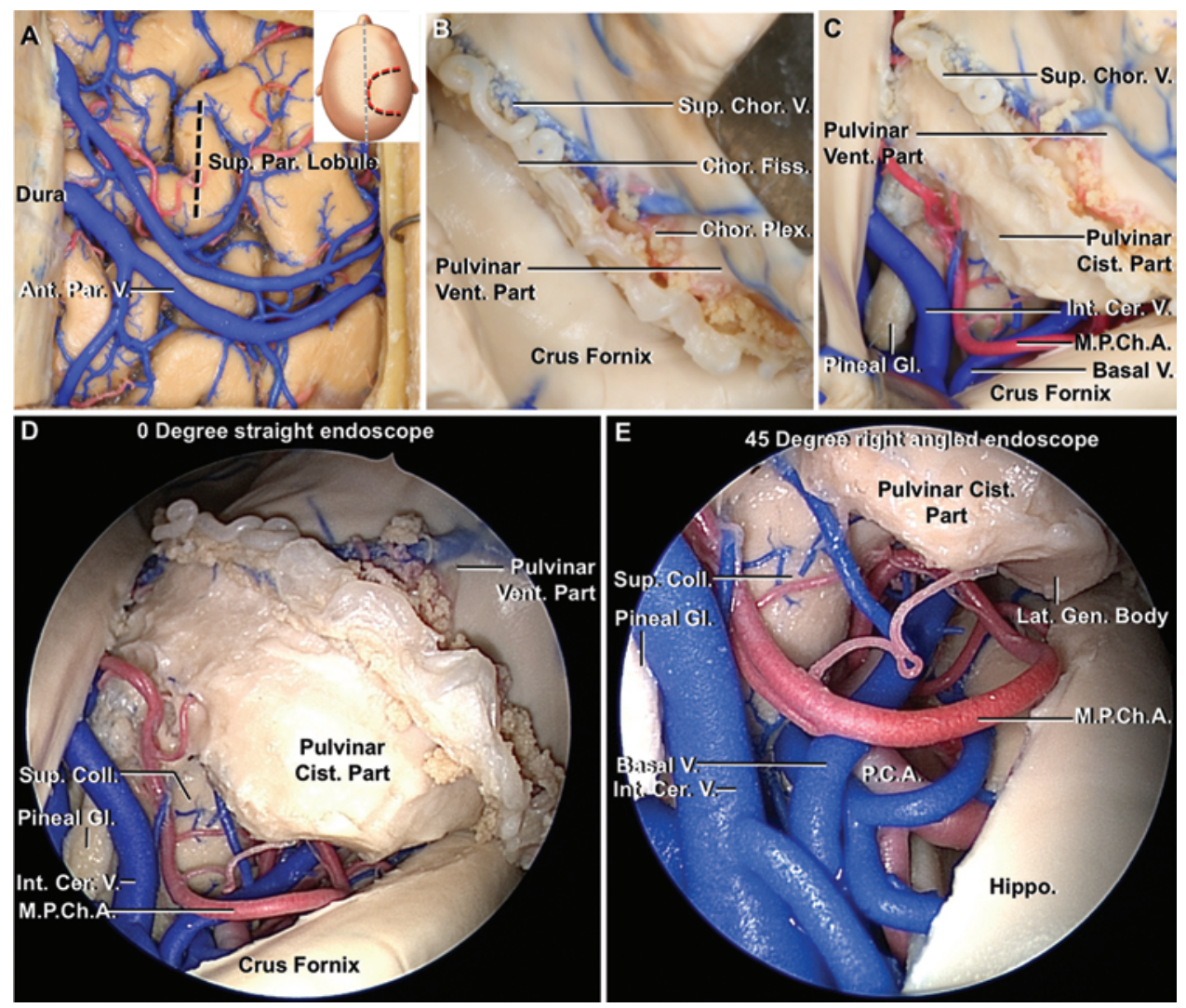

FIG. 4. Superior parietal lobule approach. A: The inset shows the scalp incision (red curved line). The cortical incision crosses the superior parietal lobe (black dotted line). B: Microscopic view in the lateral ventricle. The lateral edge of the crus of the fornix, the choroidal fissure, and the choroid plexus cross the medial edge of the ventricular part of the pulvinar. The cisternal part of the pulvinar is hidden below the crus fornix. C: Opening the choroidal fissure and retracting the fornix medially in the microscopic view exposes the cisternal surface of the pulvinar. The pineal gland, superior colliculus, and medial posterior choroidal artery have been exposed in the quadrigeminal cistern. D: $0^{\circ}$ endoscopic view after opening the choroidal fissure. The superior and medial surfaces of the cisternal part of the pulvinar, internal cerebral veins, medial posterior choroidal artery, and pineal gland have been exposed. E: The $45^{\circ}$ endoscope is directed laterally through the choroidal fissure and below the pulvinar toward the lateral geniculate body and superior colliculus. Ant. = anterior; Cer. = cerebral; Chor. = choroid, choroidal; Cist. = cisternal; Coll. = colliculus; Fiss. = fissure; Gen. = geniculate; GI. = Gland; Hippo. = hippocampus; Int. = internal; Lat. = lateral; M.P.Ch.A. = medial posterior choroidal artery; Par. = parietal; P.C.A. = posterior cerebral artery; Plex. = plexus; Sup. = superior; V. = vein; Vent. = ventricular.

segments of the posterior cerebral and medial posterior choroidal arteries, pineal gland, and quadrigeminal and posterior ambient cisterns are exposed (Fig. 4C). It was difficult to expose the contralateral internal cerebral vein, trochlear nerve, tentorial edge, and lateral geniculate body through this approach. The microscope provided satisfactory exposure of the ventricular and superior and lateral cisternal surfaces of the pulvinar. The velum interpositum forming the roof of the third ventricle and through which the internal cerebral veins and medial posterior choroidal arteries course may be exposed anterior to the pineal gland. The microscope is optimal for tumors involving the quadrigeminal cistern and suitable for tumors that extend exophytically into the cistern and if a tumor extends into the posterior body and atrium of the lateral ventricle. ${ }^{30}$

Endoscopic View. Adding the $0^{\circ}$ endoscope provided good visualization of the upper ventricular and cisternal parts of the pulvinar. Advancing the $0^{\circ}$ endoscope medially through the choroidal fissure brought into view the posterior choroidal arteries, internal cerebral vein, superi- or colliculus, and pineal gland (Fig. 4D). The contralateral internal cerebral vein could be seen without additional retraction. The tip of the $45^{\circ}$ endoscope is advanced through the choroidal fissure and along the fornix to provide good visualization of the superior colliculi in the quadrigeminal cistern. Directing the $45^{\circ}$ endoscope medially exposed the pineal gland, internal cerebral vein, and medial posterior choroidal artery and provided a better view than the microscope of the pineal gland, internal cerebral vein, superior colliculus, and pulvinar surface facing the posterior third ventricle. Directing the angled endoscope laterally provided a far better view of the quadrigeminal cistern adjacent to the choroidal fissure, particularly the posterior choroidal arteries. Directing the $45^{\circ}$ endoscope laterally below the pulvinar provided a good view of the lateral geniculate body, which is difficult to see in the microscopic view (Fig. 4E). The $0^{\circ}$ and $45^{\circ}$ endoscopes extended the view to the lateral, medial, and inferior limits of the cisternal part of the pulvinar. Although the endoscope could provide the same view as the microscope, the endoscope does have 2 advantages. One advantage is better visualiza- 

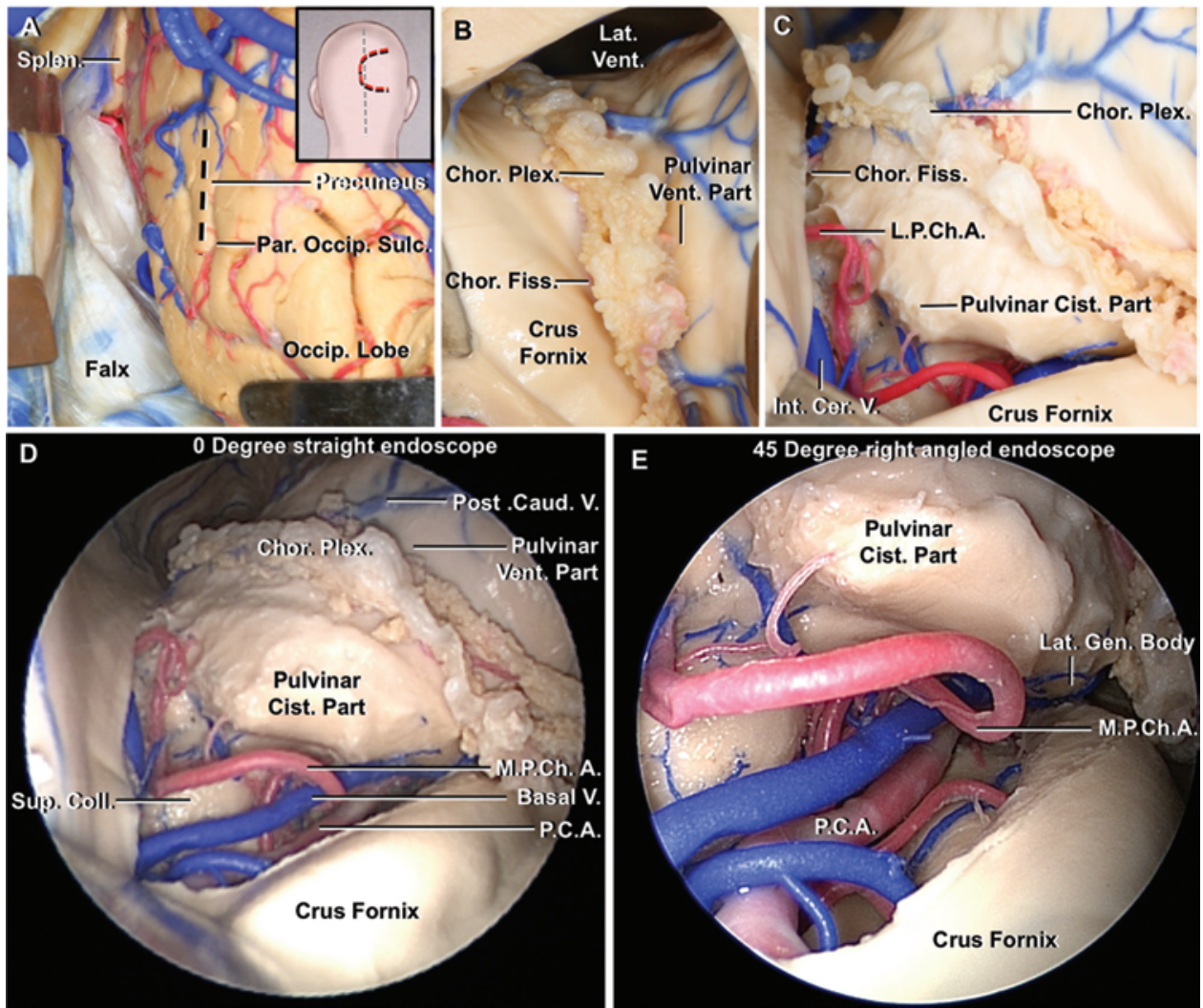

FIG. 5. Posterior interhemispheric transprecuneus approach. A: The cortical incision on the right precuneus (black dotted line) opens into the atrium. The inset shows the skin incision (red curved line). B: Microscopic view in the lateral ventricle. The crus of the fornix, choroidal fissure, choroid plexus, and ventricular part of the pulvinar have been exposed. The angle of entry into the lateral ventricle is slightly lower and is directed from medial to lateral as compared with the superior parietal lobule approach shown in Fig. 3. C: Exposure of the cisternal surface of the pulvinar and superior colliculus by opening the choroidal fissure with the microscope. D: $0^{\circ}$ endoscopic view exposing the posterior and medial surfaces of the cisternal part of the pulvinar and superior colliculus. E: The $45^{\circ}$ endoscope angled laterally exposes the lateral geniculate body at the lower anterior edge of the lateral posterior choroidal and posterior cerebral arteries. Caud. = caudate; Cer. = cerebral; Chor. = choroid, choroidal; Cist. = cisternal; Coll. = colliculus; Fiss. = fissure; Gen. = geniculate; Int. = internal; Lat. = lateral; L.P.Ch.A. = lateral posterior choroidal artery; M.P.Ch.A. $=$ medial posterior choroidal artery; Occip. = occipital; Par. Occip. = parieto-occipital; P.C.A. = posterior cerebral artery; Plex. = plexus; Post. = posterior; Splen. = splenium; Sulc. = sulcus; Sup. = superior; V. = vein; Vent. = ventricular, ventricle.

tion of the lateral and medial limits of the cisternal surface of the pulvinar. The other is control of the proximal part of the posterior choroidal arteries as they wrap around the pulvinar. Morcos and Kaibara ${ }^{24}$ reported that some lateral dorsal pulvinar arteriovenous malformations have choroidal feeders. The entire ventricular part could be seen with both the microscope and endoscope.

\section{Posterior Interhemispheric Transprecuneus Approach}

The posterior interhemispheric transprecuneus approach opens through the precuneus on the medial side of the occipital lobe (Fig. 5A) and enters the medial wall of the atrium at a slightly lower and more posterior level than the approach through the superior parietal lobule. It requires opening the choroidal fissure to expose the adjacent cisternal surface of the pulvinar. ${ }^{14,17}$ The ventricular surface of the pulvinar may also be exposed before opening into the atrium since this approach is directed along the medial surface of the hemisphere. In this approach, the precuneus is allowed to settle away from the falx cerebri if the side of the lesion is placed downward. This approach may require additional retraction of the medial occipital lobe and provides a narrow surgical corridor and angle of approach. An alternative to minimize the need for retraction is to place the pulvinar in the dependent position so that the ipsilateral occipital lobe settles away from the falx cerebri without retraction as CSF is removed. The endoscopic trajectory is directed through the cortical incision on the surface of the precuneus into the posterior body and atrium as in the microscopic approach. However, for the endoscopic approach, additional brain retraction is not needed once the endoscope enters the atrium.

Microscopic View. The first structures exposed after opening through the precuneus are the atrial surface of the pulvinar and the structures along the choroidal fissure (Fig. 5B). Opening the choroidal fissure from the atrium exposes the upper and adjacent posterior cisternal surface of the pulvinar, but exposure of the inferior and medial surface is limited due to the medial to lateral direction of the exposure (Fig. 5C). In this approach through the interhemispheric fissure, the cisternal surface may also be exposed before opening through the medial wall of the 


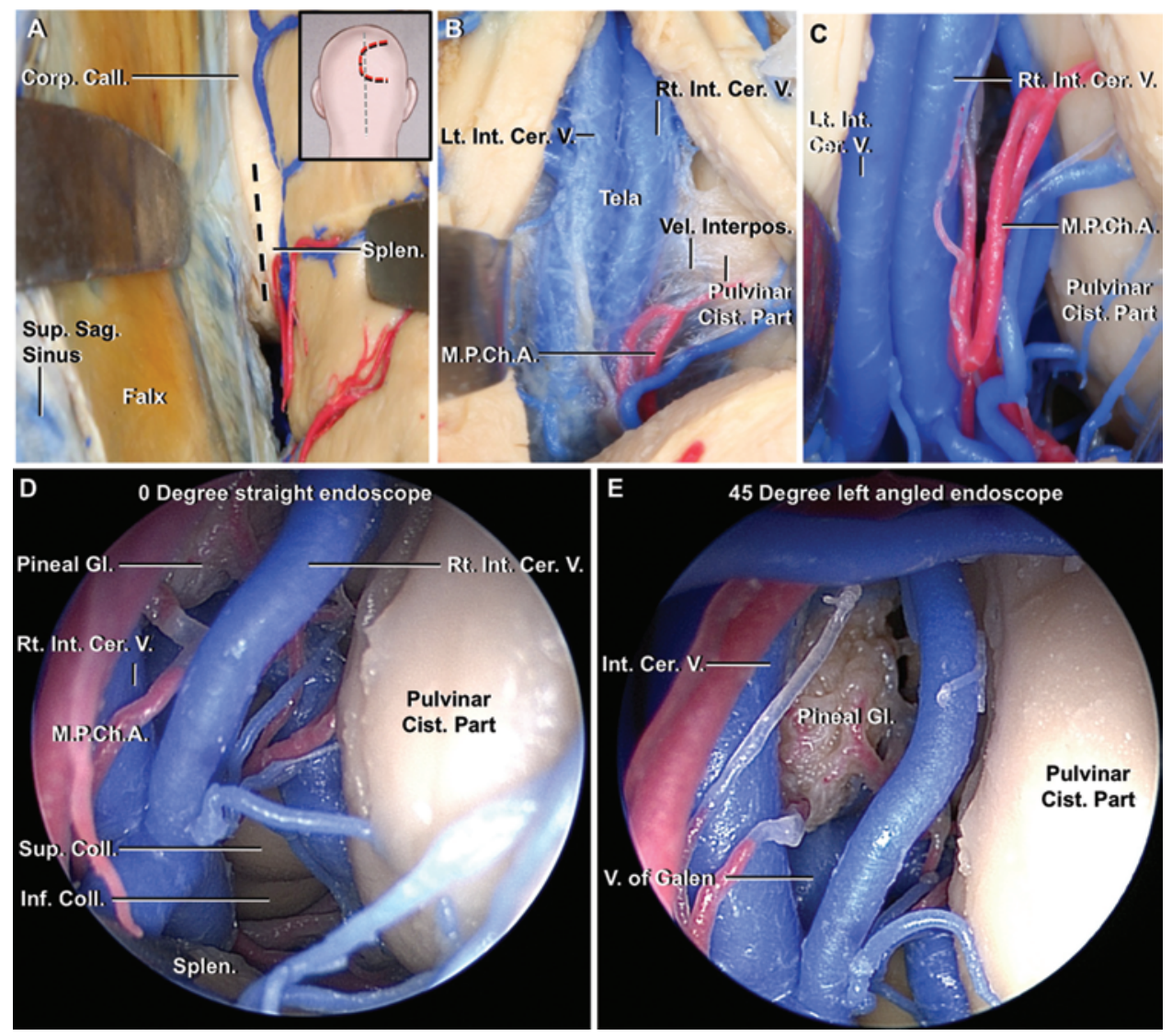

FIG. 6. Posterior interhemispheric transcallosal approach (interhemispheric velum interpositum approach). A: The splenium is opened in the midline (black dotted line). The scalp incision (red curved line) is shown in the inset. B: The velum interpositum, internal cerebral veins, medial posterior choroidal artery, and cisternal part of the pulvinar can be seen through the tela choroidea. C: The tela has been removed. $\mathrm{D}: \mathrm{A} 0^{\circ}$ endoscopic view with the right internal cerebral vein retracted medially. $\mathrm{E}:$ The $45^{\circ}$ endoscope, angled medially, exposes the pineal gland between the internal cerebral veins. Call. = callosum; Cer. = cerebral; Cist. = cisternal; Coll. = colliculus; Corp.= corpus; GI. = gland; Inf. = inferior; Int. = internal; Interpos. = interpositum; Lt. = left; M.P.Ch.A. = medial posterior choroidal artery; Rt. = right; Sag. = sagittal; Splen. = splenium; Sup. = superior; V. = vein; Vel. = velum.

atrium. The ipsilateral internal cerebral vein, superior colliculus, and medial posterior choroidal artery could be seen. It was difficult to see the third ventricle, trochlear nerve, and lateral geniculate body. The transventricular access provides only limited access to the structures in the ambient and quadrigeminal cisterns even with opening of the choroidal fissure or the adjacent medial surface of the hemisphere, because of the medial to lateral trajectory of the approach. The main disadvantage of the microscope is that it provides a narrower access and angle of approach than the endoscope. ${ }^{16,17,22}$

Endoscopic View. The $0^{\circ}$ endoscope provided good visualization of the posterior body and atrial and adjacent cisternal surfaces of the pulvinar (Fig. 5D). Advancing the $0^{\circ}$ endoscope through the choroidal fissure from the atrium exposed the pineal gland, medial posterior choroidal artery, posterior cerebral artery, and internal cerebral vein (Fig. 5D). Directing the $45^{\circ}$ endoscope medially added the superior colliculus to the exposure. Directing the $45^{\circ}$ endoscope laterally below the pulvinar exposed the lateral geniculate body and provided access for partial proximal control of the posterior cerebral and posterior choroidal arteries in the ambient and quadrigeminal cisterns in deal- ing with vascular lesions (Fig. 5E). Both the $0^{\circ}$ and $45^{\circ}$ endoscopes provided excellent views of the lateral and medial surfaces of the upper cisternal part.

\section{Posterior Interhemispheric Transcallosal Approaches}

The posterior interhemispheric transcallosal approach provides 3 routes to the pulvinar. Two are directed through the corpus callosum and one to the lateral ventricle. The splenium sits both above and posterior to the pulvinar. One route is anterior to the posterior edge of the splenium through the roof of the quadrigeminal cistern to the upper surface of the cisternal part of the pulvinar, and the other is along a lower trajectory through the posterior edge of the splenium to the posterior surface of the cisternal part of the pulvinar. The third approach is directed lateral to the ventricular surface of the pulvinar. The incision in the splenium for this approach is directed through the junction of the cingulate or parahippocampal gyri near or just medial to the isthmus. The posterior transcallosal route offers both a medial route to the cisternal surface of the pulvinar (Fig. 6) and a more lateral route directed to the posterior body and atrium of the lateral ventricle (see Fig. 8). One route, the interhemispheric velum interpositum approach, 

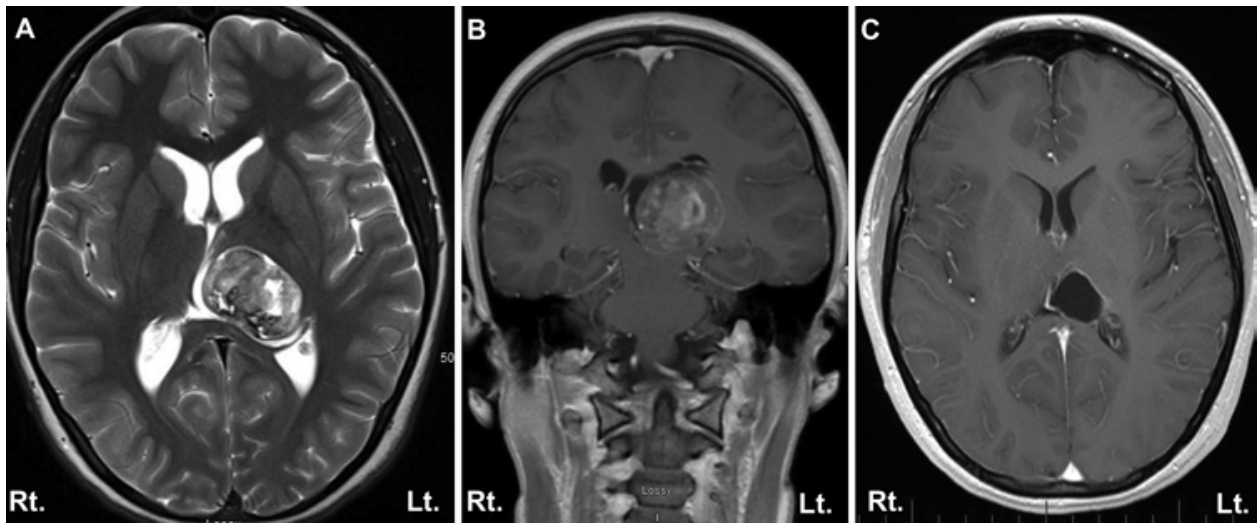

FIG. 7. Illustrative case. A: Axial T2-weighted image showing a posterior thalamic tumor including the ventral and cisternal parts of the pulvinar. There is no hydrocephalus. B: Coronal T1-weighted contrast-enhanced image. C: Axial T1-weighted contrastenhanced image showing no evidence of recurrence at the 5-year follow-up.

crosses the velum interpositum to reach the cisternal part of the pulvinar from the medial side, ${ }^{20}$ while 2 other routes pass through the posterior edge of the splenium and cingulate gyrus, respectively, to initially reach the ventricular part from the lateral side.

The microscope is suited for superior and medial pulvinar lesions like cavernous malformations that reach the cisternal surface ${ }^{6}$ and for lesions that extend into the atrium or third ventricle or upward through the splenium or medially under the corpus callosum., ${ }^{2,14}$ One of the advantages of the velum interpositum approach with the microscope is the ease of approaching lesions extending into the third ventricle. ${ }^{20}$

Interhemispheric Velum Interpositum Approach, Medial to Lateral. This approach through the splenial roof above the pulvinar exposes the superior and medial portions of the upper cisternal surface. In the three-quarter prone position with the parietal region placed most superiorly, the dura mater is reflected toward the superior sagittal sinus. The splenium of the corpus callosum is incised slightly off the midline toward the side of the pulvinar harboring the lesion to open the velum interpositum and expose the internal cerebral vein (Fig. 6A and B). The cisternal surface of the pulvinar and choroidal fissure are exposed along the lateral edge of the internal cerebral vein in the velum interpositum. Opening the choroidal fissure is difficult from the cisternal side and requires extensive retraction to expose the atrial surface of the pulvinar. This approach to the cisternal surface is appropriate for patients without hydrocephalus because the operation can be easily performed from the midline. ${ }^{27}$

Microscopic View. After opening the corpus callosum, the first structures seen are the internal cerebral veins covered with tela choroidea. Next, the trajectory of the microscope is directed slightly laterally. After lateral retraction of the splenium and cutting the tela choroidea, the medial part of the cisternal pulvinar could be seen laterally. The choroidal fissure crosses the lateral edge of this exposure along with the edge of the internal cerebral vein (Fig. 6C). The posterior choroidal arteries, pineal gland, internal cerebral veins, and medial and superior surfaces of the pulvinar are seen. After gentle retraction of the internal cerebral veins, the pineal gland is easily seen between the internal cerebral veins, but it is difficult to see the structures in the lower part of the quadrigeminal cistern, such as the trochlear nerve, tentorial edge, lateral geniculate body, and superior colliculus.

Endoscopic View. The endoscope, following the same trajectory as the microscope, provides an improved view of the same structures as seen with the microscope. Advancing the $0^{\circ}$ endoscope provided a better visualization of relationships along the medial cisternal surface of the pulvinar, galenic complex, superior and inferior colliculi, and pineal gland than was provided by the microscope (Fig. 6D). Directing the $45^{\circ}$ endoscope medially and parallel to the splenial incision and placing the tip of the endoscope on the level of the lower edge of the splenium exposed the relationship between the pineal gland and the galenic venous complex (Fig. 6E). Retraction of the fornix from medial to lateral exposed the superior and medial surfaces of the pulvinar and the medial posterior choroidal artery, which passes around the posteromedial surface of the pulvinar in the quadrigeminal cistern.

The endoscope adds exposure of the junction of the pulvinar with and including the superior and inferior colliculi that is not possible with the microscope. Both the microscope and the endoscope are useful in the absence of hydrocephalus because there is no need for corticotomy, reducing the risk of seizure and visual or language disturbance. A disadvantage is the risk of a disconnection syndrome because of the incision into the splenium. As nearly the same structures could be seen in each exposure, the route should be selected depending on the location of the target.

Illustrative Case. A 13-year-old girl presented with headaches. MR imaging demonstrated a posterior thalamic tumor affecting the pulvinar (Fig. 7A and B). Grosstotal tumor resection was achieved through a left parietal craniotomy, posterior interhemispheric transcallosal-paravenous approach (interhemispheric velum interpositum route). The pathology was consistent with diffuse infiltrative Grade 2 astrocytoma. During her hospital stay, she had right hemiparesis that steadily improved and a single postoperative seizure. She received no adjuvant therapy. She is now 5 years from surgery with no evidence of tumor recurrence (Fig. 7C). 

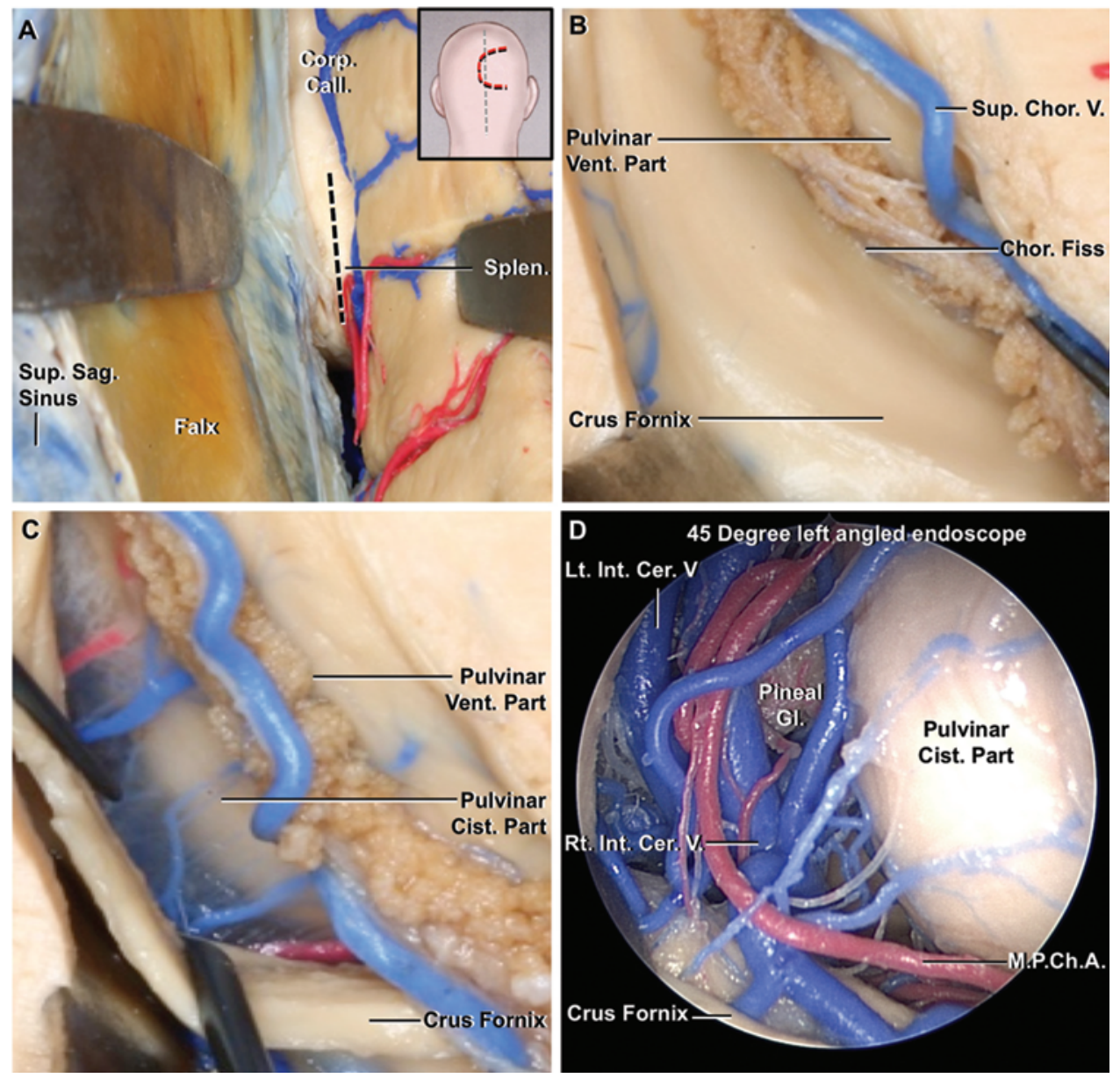

FIG. 8. Posterior interhemispheric transcallosal approach (lateral transcallosal approach to the ventricle). A: The cingulate gyrus and splenium have been opened to the right of the midline (black dotted line). The inset shows the skin incision (red curved line). B: Microscopic view. The choroidal fissure, crus of fornix, and ventricular part of the pulvinar are exposed. The cisternal part of the pulvinar is hidden below the crus of the fornix. C: Enlarged microscopic view. The cisternal part of the pulvinar has been exposed by opening the choroidal fissure. D: Advancing the $0^{\circ}$ endoscope through the choroidal fissure exposes the internal cerebral vein, pineal gland, medial posterior choroidal artery, and cisternal part of the pulvinar. Both the superior and inferior colliculi are exposed. Call. = callosum; Cer. = cerebral; Chor. = choroidal; Cist. = cisternal; Corp. = corpus; Fiss. = fissure; GI. = gland; Int. = internal; Lt. = left; M.P.Ch.A. = medial posterior choroidal artery; Rt. = right; Sag. = sagittal; Splen. = splenium; Sup. = superior; $\mathrm{V}$. = vein; Vent. = ventricular.

Lateral Transcallosal Approach to the Ventricle, Lateral to Medial. This approach is directed laterally through the splenium to the lateral ventricle and exposes the posterior body and atrium of the lateral ventricle. The head position and interhemispheric dissection are the same as in the former route (Fig. 8A). The splenium of the corpus callosum is incised $2 \mathrm{~cm}$ obliquely from the midline on the side of the lesion to open the lateral ventricle. The ventricular surface of the pulvinar and choroidal fissure are exposed. Opening the choroidal fissure requires extensive retraction and exposes the cisternal surface of the pulvinar.

Microscopic View. After opening the corpus callosum, the first structures seen in the lateral ventricle are the choroid plexus, the fornix, and the ventricular part of the pulvinar (Fig. 8B). Lateral retraction of the splenium and medial mobilization of the fornix are required to visualize the deep structures. Opening the choroidal fissure from the atrium exposes the internal cerebral vein, the medial posterior choroidal artery, and the cisternal part of the pulvinar (Fig. 8C). In this approach, both parts of the pulvinar can be seen concurrently more easily than in the interhemispheric velum interpositum approach. The medial and superior aspects of the cisternal part of the pulvinar are exposed. It is difficult to see the structures in the lower part of the quadrigeminal cistern because of the numerous vessels obstructing the view.

Endoscopic View. The endoscope provides an improved view of the same structures seen with the microscope. Advancing the $0^{\circ}$ endoscope provides a better exposure of relationships along the medial cisternal surface of the pulvinar, the internal cerebral vein, and the superior and inferior colliculi than that provided by the microscope. Directing the $45^{\circ}$ endoscope medially provides a wider and better visualization of the relationship between the pineal gland, the galenic venous complex, and the cisternal part of the pulvinar (Fig. 8D). 

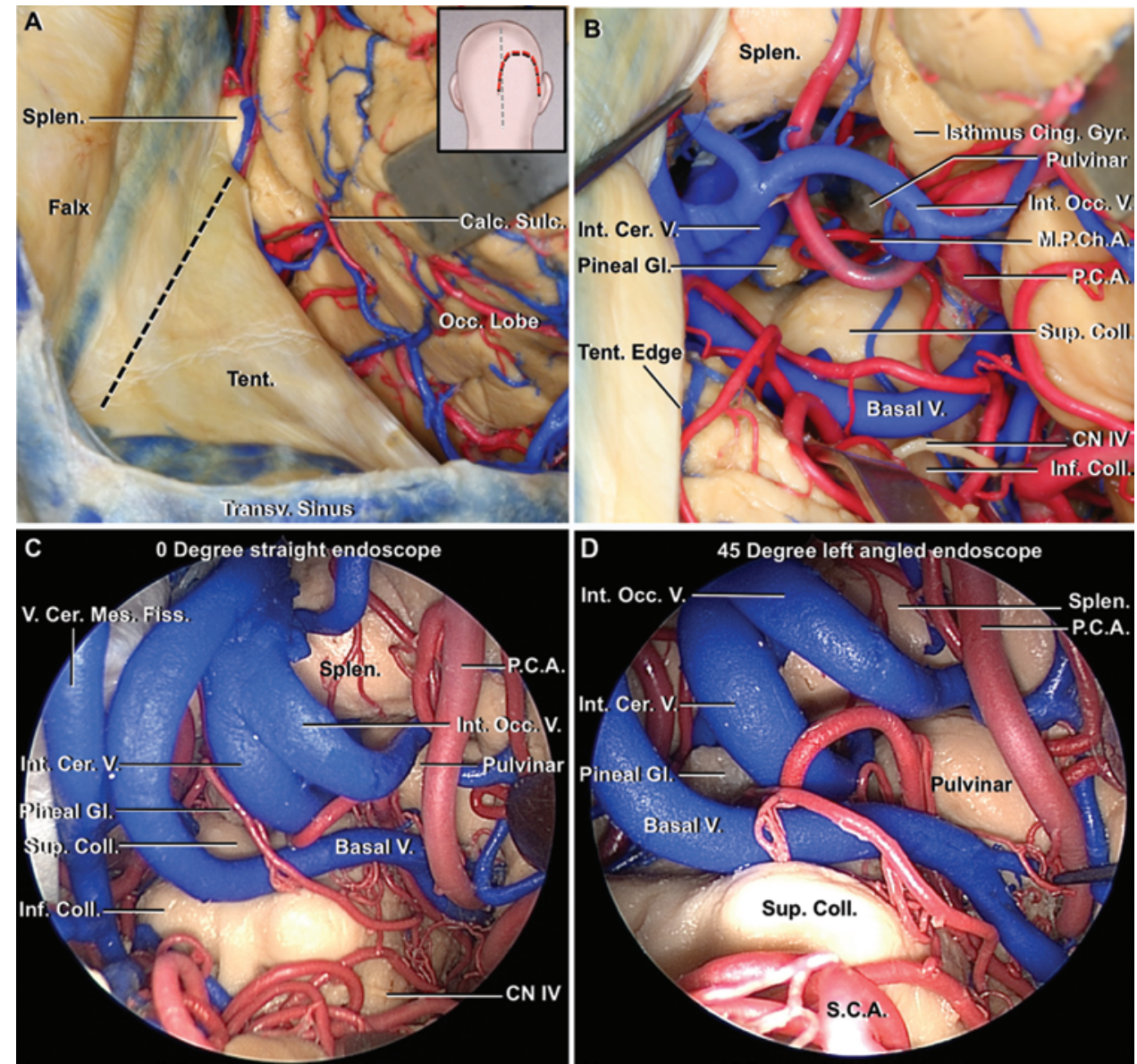

FIG. 9. Posterior interhemispheric transtentorial approach. A: The skin incision (red curved line in inset) and right tentorial incision (black dotted line) are shown. B: Microscopic view. The inferomedial cisternal surface of the pulvinar is exposed deep to the splenium and at the anterior edge of the isthmus of the cingulate gyrus. The superior colliculus and trochlear nerve have also been exposed. C and D: $0^{\circ}$ endoscopic views. The $0^{\circ}$ endoscope provides a view of the pulvinar and the veins and arteries in the quadrigeminal cistern $(\mathrm{C})$. The $45^{\circ}$ endoscope has been directed medially to show the structures in the quadrigeminal cistern (D). Calc. = calcarine; Cer. = cerebral; Cer. Mes. = cerebellomesencephalic; Cing. = cingulate; Coll. = colliculi; Fiss. = fissure; GI. = gland; Gyr. = gyrus; Inf. = inferior; Int. = internal; M.P.Ch.A. = medial posterior choroidal artery; Occ. = occipital; P.C.A. = posterior cerebral artery; S.C.A. = superior cerebellar artery; Splen. = splenium; Sulc. = sulcus; Sup. = superior; Tent. = tentorial, tentorium; Transv. = transverse; $\mathrm{V}$. = vein .

\section{Posterior Interhemispheric Transtentorial Approach}

This approach, along the occipital lobe and interhemispheric fissure with opening of the tentorium $1 \mathrm{~cm}$ lateral and parallel to the straight sinus (Fig. 9A), exposes the inferior and medial cisternal surface of the pulvinar but does not access the ventricular surface. It provides access to lesions extending into the ambient cistern and floor of the quadrigeminal cistern at the level of the trochlear nerve but not the contralateral quadrigeminal cistern. The operation is performed in the three-quarter prone position ${ }^{3}$ with the head elevated so as to leave the sinus confluence slightly above the atrium. The occipital surface on the side of the lesion is placed downward to allow it to relax away from the falx cerebri, thus reducing the need for retraction. ${ }^{10,30}$ The dura is cut parallel to and $1 \mathrm{~cm}$ away from the straight sinus. The thick arachnoid around the ipsilateral basal vein of Rosenthal is divided, and the approach is directed below the splenium to the pulvinar harboring the lesion.
Microscopic View. After the dura mater is reflected toward the straight sagittal sinus and the arachnoid membranes have been dissected, the first structure encountered in the depth of the interhemispheric fissure is the internal occipital vein. It passes from the anterior part of the medial calcarine cortex to the vein of Galen and often blocks access to the pulvinar, but can be displaced and preserved in some cases. The posterior and medial surfaces of the pulvinar and the choroidal arteries on their way to the choroidal fissure and choroid plexus are exposed. The exposure can be directed laterally along the midbrain to the ambient cistern to expose the lateral margin of the cerebral peduncle and the posterior cerebral artery. The trochlear nerve, superior colliculus, posterior cerebral artery, and internal cerebral and basal veins on the ipsilateral side were exposed. Although the area above the vein of Galen is exposed, it is difficult to see the contralateral pulvinar and contralateral part of the quadrigeminal cistern (Fig. 9B). Disadvantages of the microscope are the need for retrac- 
tion of the occipital lobe adjacent the tentorium at or near the calcarine sulcus, which could lead to homonymous hemianopia, and the possible need to sacrifice bridging veins, including the galenic venous complex, to expand the exposure along the quadrigeminal cistern. ${ }^{25,35}$ It would be difficult to bring lesions lateral to the pulvinar into the operative field.

Endoscopic View. The direction of the endoscope is parallel and slightly lateral to the straight sinus. Advancing the $0^{\circ}$ endoscope under the tentorium cerebelli and straight sinus exposed part of the contralateral quadrigeminal cistern and pulvinar behind the internal occipital vein and galenic complex (Fig. 9C). The tip of the $45^{\circ}$ endoscope is placed parallel to the straight sinus just before the galenic venous complex and turned laterally. Directing the $45^{\circ}$ endoscope contralaterally provided a better view of the relationships around the contralateral pulvinar than the microscope (Fig. 9D). The endoscope reduced the need for retraction of the occipital lobe and provided a better and wider view of the posterior, inferior, and medial surfaces of the pulvinar than the microscope. There is a risk of air embolism in this approach ${ }^{11}$ if the sitting position is selected, and of morbidity related to sacrifice of bridging veins draining into the tentorial sinuses and galenic complex. ${ }^{47}$

\section{Supracerebellar Infratentorial Approach}

The supracerebellar infratentorial approach provides access to the inferior and medial cisternal surfaces of the pulvinar and pineal gland and lower surface of the splenium, superior cerebellar peduncle, and trochlear nerve, but does not access the ventricular part of the pulvinar. In the three-quarter prone or sitting position, a vertical midline skin incision is made and a suboccipital craniectomy performed, ${ }^{30}$ the dura mater opened, the vermis of the cerebellum retracted inferiorly, and the thick arachnoid membrane covering the quadrigeminal cistern divided to define the galenic venous complex (Fig. 10A). The approach continues along the undersurface of the tentorium cerebelli to the lower part of the quadrigeminal and adjacent part of the ambient cisterns. The advantage is that this approach allows easy orientation without damaging important structures.

Microsurgical View. The internal occipital and cerebral and basal veins and the vein of the cerebellomesencephalic fissure are exposed. The pulvinar is located above the internal occipital vein, which exits the anterior end of the calcarine sulcus at the level of the lower edge of the pulvinar (Fig. 10A). If the pulvinar is not visible, opening the tentorium cerebelli may bring it into view and allow access to arteriovenous malformations by branches of the posterior cerebral artery in the upper third of the operative field. ${ }^{11,32}$ The vascular structures exposed include the posterior cerebral, a tentorial branch of the superior cerebellar, and the medial posterior choroidal arteries, vein of Galen, and internal occipital and cerebral veins. The floor of the ambient cistern is the middle cerebellar peduncle and the medial wall is the midbrain along the lateral mesencephalic sulcus. The lower margin of the operative field is the superior colliculus, and the lateral margin is the cisternal surface of the pulvinar. Dividing the tentorium cer- ebelli and elevating the parahippocampal gyrus increases the upward exposure of the pulvinar. When venous structures block access to the pulvinar, it is possible to retract them medially. Disadvantages of the microscope are the possible need to sacrifice bridging veins and reduced access to the pulvinar by the internal cerebral vein.

Endoscopic View. In this approach, as the range of the working space is wider than in other approaches, the surgeon can choose the best position to obtain the necessary operative view. Inserting the $0^{\circ}$ endoscope along the left tentorium cerebelli or the parahippocampal gyrus and elevating the parahippocampal gyrus provides added access to the posterior surface of the pulvinar, but the superior cerebellar artery may block the view (Fig. 10B and C). The $45^{\circ}$ endoscope with retraction of the superior cerebellar artery provides a better view of the venous complex and pulvinar than the microscopic view (Fig. 10D). Advancing the $45^{\circ}$ endoscope laterally provided a better view of the medial and inferior pulvinar than the microscopic view, especially when the internal occipital vein or the straight sinus blocked the view of the microscope (Fig. 10D). The $45^{\circ}$ endoscope provided a better view of the pulvinar than the $0^{\circ}$ endoscope.

\section{Discussion}

Tumors in the pulvinar can present as either diffuse or circumscribed lesions. Microsurgical removal may be indicated for circumscribed lesions. ${ }^{45}$ These lesions may also grow exophytically into the lateral and third ventricles, making them suited for a transventricular approach..$^{30}$ Pulvinar tumors sometimes grow posteriorly or inferiorly, extending into the midbrain and ipsilateral half of the quadrigeminal and ambient cisterns. ${ }^{44}$ In these cases, the posterior interhemispheric transtentorial approach and supracerebellar infratentorial approach may provide satisfactory access for biopsy and selective removal.

Five approaches to the surface of the pulvinar using both the microscope and the endoscope were examined. Tables 1 and 2 list the structures and surfaces of the pulvinar accessed by each approach. Potential complications related to each approach are listed in Table 3.14,27,29,35

The superior parietal lobule, interhemispheric transprecuneus, and lateral transcallosal approaches open directly into the ventricle but at slightly different angles, and all 3 provide access through the choroidal fissure or posterior to the fornix to the adjacent cisternal part. The superior parietal lobule approach provides the most anterior access with the transprecuneus approach entering the ventricle slightly posterior to the superior parietal lobule approach. The transcallosal approach offers 2 routes to the ventricular surface of the pulvinar. One is through the corpus callosum anterior to the posterior edge of the splenium, a more superior approach that exposes the posterosuperior ventricular surface of the pulvinar. The other route, a more posterior approach, is directed through the posterior edge of the splenium, which crosses the midline posterior to the pulvinar. Opening the posterior edge of the splenium offers a more posterior and inferior approach to the cisternal surface of the pulvinar.

In opening the choroidal fissure from the ventricle to 

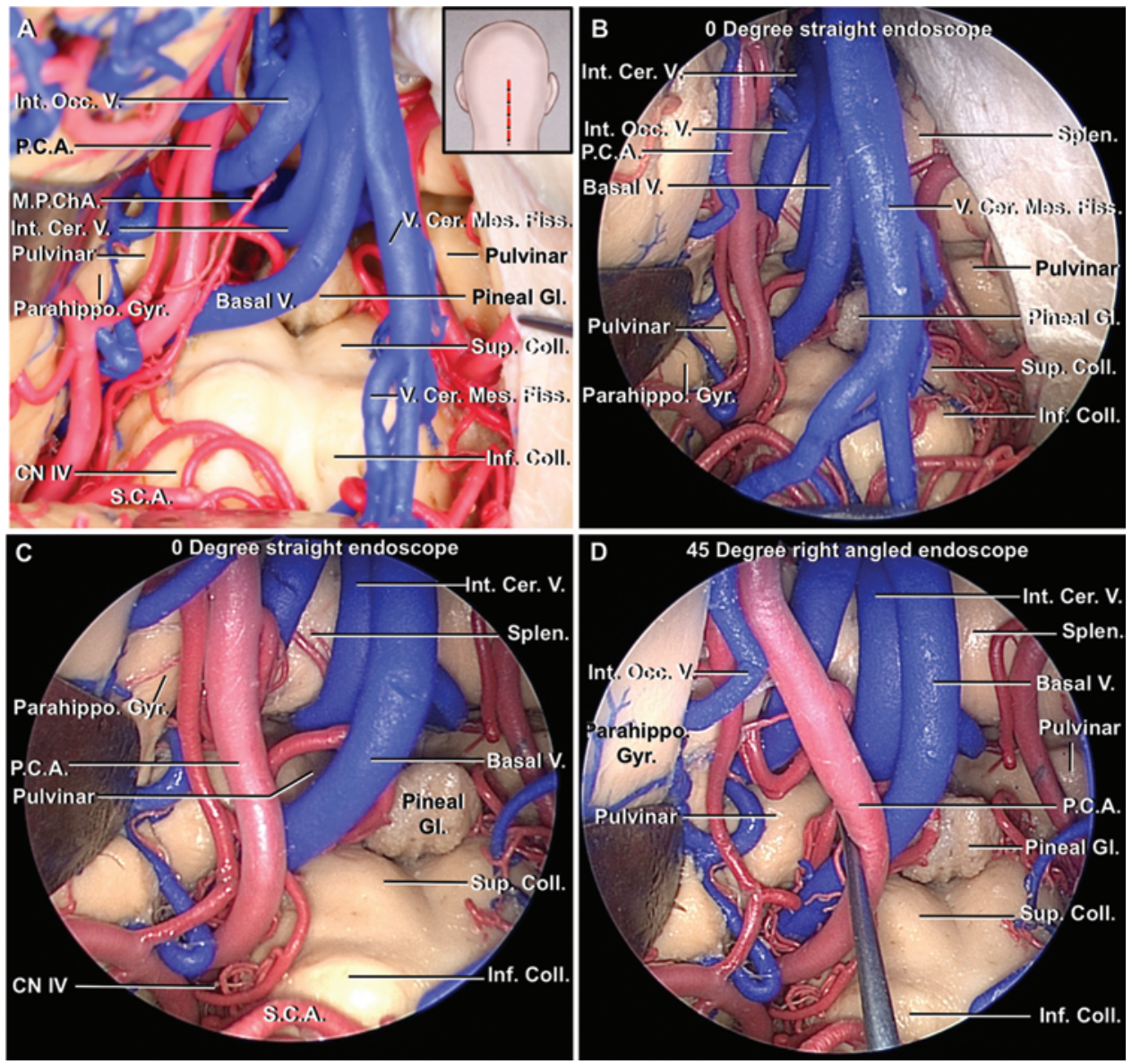

FIG. 10. Supracerebellar infratentorial approach. A: The venous complex exposed emptying into the vein of Galen includes the internal occipital, basal, and internal cerebral veins, which nearly hide the cisternal surface of the pulvinars. The left pulvinar cannot be seen without retraction. The inset shows the midline skin incision (red dotted line). The left half of the tentorium has been opened and the left parahippocampal gyrus has been retracted to improve exposure of the left pulvinar. The pulvinar is at the lateral margin of the operative field. $\mathrm{B}: 0^{\circ}$ endoscopic view with retraction of the left isthmus of the cingulate and parahippocampal gyri. C: $0^{\circ}$ endoscopic view with retraction of the parahippocampal gyrus and the posterior cerebral artery to expose the posterior and inferior surface of the pulvinar. D: Right $45^{\circ}$ endoscopic view directed to the left with retraction of the parahippocampal gyrus. The inferior and medial aspects of the pulvinar have been exposed in the quadrigeminal cistern. Relationships between the pulvinar and internal occipital vein and the superior cerebellar artery, which interrupts the view. Cer. $=$ cerebral; Cer. Mes. = cerebellomesencephalic; Coll. = colliculus; Fiss. = fissure; GI. = gland; Gyr. = gyrus; Inf. = inferior; Int. = internal; M.P.Ch.A. $=$ medial posterior choroidal artery; Occ.= occipital; Parahippo. = parahippocampal; P.C.A. = posterior cerebral artery; S.C.A. = superior cerebellar artery; Splen. = splenium; Sup. = superior; V. = vein.

access the cisternal part of the pulvinar, the superior parietal lobule approach offers a more lateral to medial trajectory for opening the choroidal fissure, and a more favorable one for exposing the cisternal surface of the pulvinar.
The transprecuneus and transcallosal approaches provide a more medial to lateral trajectory, which is less favorable for exposing the cisternal surface of the pulvinar through the choroidal fissure. The $0^{\circ}$ endoscope provided more

TABLE 1. Summary of the exposable structures around the pulvinar

\begin{tabular}{ccccccccc}
\hline Approach & LGB & Pineal & Sup Colliculus & Inf Colliculus & Trochlear Nerve & PCA & PChAs & SCA \\
\hline SPLA & E & EM & EM & & & EM & EM & E \\
\hline PPA & E & & EM & & & EM & EM \\
\hline PTC & & EM & E & E & EM & EM & EM & EM \\
\hline PTT & & EM & EM & EM & EM & EM & EM & EM \\
\hline SCIT & & EM & EM & EM
\end{tabular}

$\mathrm{E}$ = endoscope only; $\mathrm{EM}$ = endoscope and microscope; inf = inferior; $\mathrm{LGB}$ = lateral geniculate body; $\mathrm{PCA}$ = posterior cerebral artery; $\mathrm{PChA}=$ posterior choroidal artery; $\mathrm{PPA}=$ posterior interhemispheric transprecuneus approach; $\mathrm{PTC}=$ posterior interhemispheric transcallosal approach; $\mathrm{PTT}=$ posterior interhemispheric transtentorial approach; SCA = superior cerebellar artery; SCIT = supracerebellar infratentorial approach; SPLA = superior parietal lobule approach; sup = superior. 
TABLE 2. The visible parts of the pulvinar in each approach

\begin{tabular}{ccccccc}
\hline & Ventricular & \multicolumn{5}{c}{ Cisternal Part of Pulvinar } \\
\cline { 3 - 7 } Approach & Part of Pulvinar & Superior & Lateral & Medial & Posterior & Inferior \\
\hline SPLA & EM & EM & EM & & \\
\hline PPA & EM & & EM & & EM \\
\hline PTC & EM & EM & & EM & EM & EM \\
\hline PTT & & & & EM & EM & EM \\
\hline SCIT & & & & & &
\end{tabular}

magnified but similar views to the microscope, but the $45^{\circ}$ endoscope extended the view to the areas medial and lateral below the pulvinars.

The posterior transcallosal, posterior interhemispheric transtentorial approach and supracerebellar infratentorial approach through the velum interpositum provide access to the cisternal surface of the pulvinar, but not to the ventricular surface. The posterior interhemispheric transtentorial approach and supracerebellar infratentorial approach provide access to the lower part of the cisternal surface with easier access as far forward as the lateral geniculate body. Accessing the ventricular surface of the pulvinar by these approaches may require resection of some of the isthmus of the cingulate gyrus and adjacent cingulate and parahippocampal gyri.

We mentioned numerous advantages of using the endoscope, but the major disadvantages of the endoscope are the lack of depth perception due to the 2D image and lack of backward and sideways vision, which makes it dangerous to move the endoscope in such a deep operative field. Extreme care is needed to prevent the endoscope from drifting into and injuring vessels, nerves, and soft brain tissues located out of view. ${ }^{39}$ This is an especially prominent problem with the angled endoscope, which, when advanced forward, does not show what lies straight ahead. ${ }^{5,9,28}$ Image fusion technology, carefully designed instrumentation, antifogging, and irrigation devices improve the safety of endoscopes..$^{32}$ Most studies of endoscopic surgeries for thalamic lesions are limited to the anterior part of the thalamus ${ }^{36}$ or endoscopic biopsy, ${ }^{1,33}$ but none have focused on the pulvinar. Tseng et al. ${ }^{41}$ performed an endoscopic supracerebellar infratentorial retropineal approach, and Thaher et al. ${ }^{40}$ performed a paramedian infratemporal supracerebellar key approach to pineal gland tumors close to the pulvinar. These approaches are likely suitable for lesions in the pulvinar as well.

The supratentorial approaches include the superior parietal lobule, ${ }^{4,18,38,42}$ posterior interhemispheric transprecuneus ${ }^{46}$ and posterior interhemispheric transcallosal approaches, ${ }^{2,12}$ which expose the ventricular part and the upper cisternal part of the pulvinar, and the occipital interhemispheric transtentorial approach, ${ }^{15}$ which exposes the lower cisternal part (Fig. 9). The supracerebellar infratentorial approach exposes the lower cisternal part of the pulvinar (Fig. 10). ${ }^{37}$ Although the superior parietal lobule and posterior interhemispheric transprecuneus approaches open directly into the atrium, they can be extended to the cisternal part by opening the choroidal fissure or the medial surface of the occipital lobe behind the fissure or by dividing the fornix unilaterally. ${ }^{14}$

\section{Conclusions}

Precise anatomical knowledge is required for treatment of pathology in the pulvinar because the operative fields obtained with different approaches differ significantly. The microscope provides a satisfactory view of the posterior surface of the pulvinar and adjacent neural and vascular structures, but the visualization of deep structures is often reduced by overhanging veins, the crus of the fornix, and space limitations. The angled endoscope, with its ability to see around corners and behind structures, facilitated exposure of the lateral geniculate body, midbrain, and inferomedial surfaces of the pulvinar. The endoscope also provides a view of the arteries and structures around the pulvinar that the microscope cannot see, including the trochlear nerve in the superior parietal lobule approach, the pineal gland in the posterior interhemispheric

TABLE 3. A summary of advantages, disadvantages, and complications of the described approaches

\begin{tabular}{|c|c|c|c|}
\hline Approach & Advantage & Disadvantage & Complication \\
\hline SPLA & Avoids the optic radiations \& language region & Long surgical distance; difficult access w/o hydrocephalus & Seizure; visual disturbance \\
\hline PPA & Low incidence of visual dysfunction & $\begin{array}{l}\text { Wider brain retraction \& narrower surgical corridor \& angle } \\
\text { of approach }\end{array}$ & Lower-extremity weakness \\
\hline PTC & $\begin{array}{l}\text { Low incidence of postop seizure; no compli- } \\
\text { cation of speech or visual function }\end{array}$ & Incision of corpus callosum & $\begin{array}{l}\text { Risk of disconnection } \\
\text { syndrome }\end{array}$ \\
\hline PTT & Low risk of seizure & Retraction of the occipital pole, injury of bridging veins & $\begin{array}{l}\text { Visual deficit, venous } \\
\text { infarction }\end{array}$ \\
\hline SCIT & $\begin{array}{l}\text { Infratentorial route is completely extra-axial \& } \\
\text { less invasive }\end{array}$ & Difficulty of lateral access $>1 \mathrm{~cm}$ & No related complications \\
\hline
\end{tabular}


transprecuneus approach, and the inferior colliculus in the posterior interhemispheric transcallosal approach.

\section{Acknowledgments}

We wish to thank Robin Barry, MA, for her assistance with the preparation of the illustrations, and Jessica Striley, BS, for her editorial assistance. This work was supported by the University of Florida Foundation.

\section{References}

1. Ahn ES, Goumnerova L: Endoscopic biopsy of brain tumors in children: diagnostic success and utility in guiding treatment strategies. J Neurosurg Pediatr 5:255-262, 2010

2. Aida T, Abe H, Iwasaki Y, Hokin K, Tsuru M: [Transcallosal approach to para-ventricular tumors.] No Shinkei Geka 12:943-950, 1984 (Jpn)

3. Ausman JI, Malik GM, Dujovny M, Mann R: Three-quarter prone approach to the pineal-tentorial region. Surg Neurol 29:298-306, 1988

4. Beks JW, Bouma GJ, Journée HL: Tumours of the thalamic region. A retrospective study of 27 cases. Acta Neurochir (Wien) 85:125-127, 1987

5. Cappabianca P, Cavallo LM, Esposito F, de Divitiis E, Tschabitscher M: Endoscopic examination of the cerebellar pontine angle. Clin Neurol Neurosurg 104:387-391, 2002

6. Chang EF, Gabriel RA, Potts MB, Berger MS, Lawton MT: Supratentorial cavernous malformations in eloquent and deep locations: surgical approaches and outcomes. Clinical article. J Neurosurg 114:814-827, 2011

7. Cheek WR, Taveras JM: Thalamic tumors. J Neurosurg 24:505-513, 1966

8. Chen CC, Lin HL, Cho DY: Endoscopic surgery for thalamic hemorrhage: a technical note. Surg Neurol 68:438-442, 2007

9. Cheng WY, Chao SC, Shen CC: Endoscopic microvascular decompression of the hemifacial spasm. Surg Neurol 70 (Suppl 1):S1.40-S1.46, 2008

10. Chi JH, Lawton MT: Posterior interhemispheric approach: surgical technique, application to vascular lesions, and benefits of gravity retraction. Neurosurgery 59 (1 Suppl 1):ONS41-ONS49, 2006

11. Dallier F, Di Roio C: Sitting position for pineal surgery: Some anaesthetic considerations. Neurochirurgie 61:164167,2015

12. Dandy WE: An operation for the removal of pineal tumors. Surg Gynecol Obstet 33:113-119, 1921

13. Fukushima T: Endoscopic biopsy of intraventricular tumors with the use of a ventriculofiberscope. Neurosurgery 2:110113,1978

14. Ishii R, Suzuki Y, Watanabe A, Mouri Y, Ishii N, Yoshii I: Gross total removal of gliomas in the pulvinar and correlative microsurgical anatomy. Neurol Med Chir (Tokyo) 42:536-546, 2002

15. Jamieson KG: Excision of pineal tumors. J Neurosurg 35:550-553, 1971

16. Jho HD, Jho DH: Endoscopic approaches for third ventricular tumors. Oper Tech Neurosurg 6:192-199, 2003

17. Kawashima M, Li X, Rhoton AL Jr, Ulm AJ, Oka H, Fujii $\mathrm{K}$ : Surgical approaches to the atrium of the lateral ventricle: microsurgical anatomy. Surg Neurol 65:436-445, 2006

18. Kawashima M, Rhoton AL Jr, Matsushima T: Comparison of posterior approaches to the posterior incisural space: microsurgical anatomy and proposal of a new method, the occipital bi-transtentorial/falcine approach. Neurosurgery $62(6$ Suppl 3):1136-1149, 2008

19. Kelly PJ: Stereotactic biopsy and resection of thalamic astrocytomas. Neurosurgery 25:185-195, 1989
20. Kempe LG, Blaylock R: Lateral-trigonal intraventricular tumors. A new operative approach. Acta Neurochir (Wien) 35:233-242, 1976

21. King WA, Wackym PA, Sen C, Meyer GA, Shiau J, Deutsch $\mathrm{H}$ : Adjunctive use of endoscopy during posterior fossa surgery to treat cranial neuropathies. Neurosurgery 49:108116, 2001

22. Lim M, Guzman R, Kelly M, Steinberg GK: Arteriovenous malformations of the basal ganglia and thalamus, in Macdonald RL (ed): Vascular Neurosurgery. Neurosurgical Operative Atlas, ed 2. New York: Thieme, 2009, pp 153-155

23. Magnan J, Chays A, Lepetre C, Pencroffi E, Locatelli P: Surgical perspectives of endoscopy of the cerebellopontine angle. Am J Otol 15:366-370, 1994

24. Morcos JJ, Kaibara T: Subcortical arteriovenous malformations, in Connolly ES, McKhann GM II, Huang J, et al (eds): Fundamentals of Operative Techniques in Neurosurgery, ed 2. New York: Thieme, 2010

25. Nazzaro JM, Shults WT, Neuwelt EA: Neuro-ophthalmological function of patients with pineal region tumors approached transtentorially in the semisitting position. J Neurosurg 76:746-751, 1992

26. O'Donoghue GM, O’Flynn P: Endoscopic anatomy of the cerebellopontine angle. Am J Otol 14:122-125, 1993

27. Ozek MM, Türe U: Surgical approach to thalamic tumors. Childs Nerv Syst 18:450-456, 2002

28. Rak R, Sekhar LN, Stimac D, Hechl P: Endoscope-assisted microsurgery for microvascular compression syndromes. Neurosurgery 54:876-883, 2004

29. Rangel-Castilla L, Spetzler RF: The 6 thalamic regions: surgical approaches to thalamic cavernous malformations, operative results, and clinical outcomes. J Neurosurg 123:676-685, 2015

30. Rhoton AL Jr: The lateral and third ventricles. Neurosurgery 51 (4 Suppl):S207-S271, 2002

31. Rhoton AL Jr: Tentorial incisura. Neurosurgery 47 (3 Suppl):S131-S153, 2000

32. Rigamonti D (ed): Cavernous Malformations of the Nervous System, ed 1. Cambridge: Cambridge University Press, 2011, pp 168-169

33. Ritz R, Feigl GC, Schuhmann MU, Ehrhardt A, Danz S, Noell S, et al: Use of 5-ALA fluorescence guided endoscopic biopsy of a deep-seated primary malignant brain tumor. $\mathbf{J}$ Neurosurg 114:1410-1413, 2011

34. Sanai N, Mirzadeh Z, Lawton MT: Supracerebellar-supratrochlear and infratentorial-infratrochlear approaches: gravity-dependent variations of the lateral approach over the cerebellum. Neurosurgery 66 (6 Suppl Operative):264-274, 2010

35. Shirane R, Kumabe T, Yoshida Y, Su CC, Jokura H, Umezawa K, et al: Surgical treatment of posterior fossa tumors via the occipital transtentorial approach: evaluation of operative safety and results in 14 patients with anterosuperior cerebellar tumors. J Neurosurg 94:927-935, 2001

36. Sood S, Nundkumar N, Ham SD: Interhemispheric endoscopic resection of large intraventricular and thalamic tumors. J Neurosurg Pediatr 7:596-599, 2011

37. Steiger HJ, Götz C, Schmid-Elsaesser R, Stummer W: Thalamic astrocytomas: surgical anatomy and results of a pilot series using maximum microsurgical removal. Acta Neurochir (Wien) 142:1327-1337, 2000

38. Sugita K, Hongo K: Posterior transcortical approach, in Apuzzo MLJ (ed): Surgery of the Third Ventricle. Baltimore: Williams \& Wilkins, 1987, pp 557-569

39. Takemura Y, Inoue T, Morishita T, Rhoton AL Jr: Comparison of microscopic and endoscopic approaches to the cerebellopontine angle. World Neurosurg 82:427-441, 2014

40. Thaher F, Kurucz P, Fuellbier L, Bittl M, Hopf NJ: Endoscopic surgery for tumors of the pineal region via a paramedian 
infratentorial supracerebellar keyhole approach (PISKA). Neurosurg Rev 37:677-684, 2014

41. Tseng KY, Ma HI, Liu WH, Tang CT: Endoscopic supracerebellar infratentorial retropineal approach for tumor resection. World Neurosurg 77:E1-E4, 2012

42. Van Wagenen WP: A surgical approach for the removal of certain pineal tumors. Report of a case. Surg Gynecol Obstet 53:216-220, 1931

43. Yamada K, Mase M, Matsumoto T: Surgery for deeply seated arteriovenous malformation: with special reference to thalamic and striatal arteriovenous malformation. Neurol Med Chir (Tokyo) 38 Suppl:227-230, 1998

44. Yaşargil MG: Diencephalon tumors, in Microneurosurgery. New York: Thieme, 1996, Vol 4B, pp 292-293

45. Yaşargil MG: Microsurgical anatomy of the basal cisterns and vessels of the brain, diagnostic studies, general operative techniques and pathological considerations of the intracranial aneurysms, in Microneurosurgery. New York: Thieme, 1984, Vol 1, pp 53-54

46. Yaşargil MG: Parieto-occipital interhemispheric approach, in Microneurosurgery. New York: Thieme, 1996, Vol 4B, pp $56-57$

47. Yonekawa Y, Imhof HG, Taub E, Curcic M, Kaku Y, Roth
P, et al: Supracerebellar transtentorial approach to posterior temporomedial structures. J Neurosurg 94:339-345, 2001

\section{Disclosures}

The authors report no conflict of interest concerning the materials or methods used in this study or the findings specified in this paper.

\section{Author Contributions}

Conception and design: Akiyama, Matsushima, Gungor, Matsuo, Tubbs, Cohen-Gadol, Rhoton. Acquisition of data: Akiyama, Gungor, Matsuo, Rhoton. Analysis and interpretation of data: Akiyama, Rhoton. Drafting the article: Akiyama, Rhoton. Critically revising the article: all authors. Reviewed submitted version of manuscript: all authors. Approved the final version of the manuscript on behalf of all authors: Akiyama. Administrative/techni$\mathrm{cal} /$ material support: Rhoton. Study supervision: Rhoton.

\section{Correspondence}

Osamu Akiyama, Department of Neurosurgery, University of Florida, PO Box 100265, Gainesville, FL 32610-0265. email: akiyamao@juntendo.ac.jp. 